\title{
Evaluation of the generality and accuracy of a new mesh morphing procedure for the human femur
}

\author{
Lorenzo Grassi $^{\mathrm{a}}$, Najah Hraiech ${ }^{\mathrm{b}}$, Enrico Schileo ${ }^{\mathrm{a}, *}$, Mauro Ansaloni $^{\mathrm{a}}$, Michel Rochette ${ }^{\mathrm{b}}$, \\ Marco Vicecontia \\ a Laboratorio di Tecnologia Medica, Istituto Ortopedico Rizzoli, Via di Barbiano 1/10, 40136 Bologna, Italy \\ ${ }^{\mathrm{b}}$ ANSYS, Bâtiment Einstein, 11 Avenue Albert Einstein, 69100 Villeurbanne, France
}

\section{A R T I C L E I N F O}

\section{Article history:}

Received 9 February 2010

Received in revised form 6 September 2010

Accepted 24 September 2010

\section{Keywords:}

Bone biomechanics

Femur

Subject-specific finite element model

Mesh morphing

Radial basis function

\begin{abstract}
A B S T R A C T
Various papers described mesh morphing techniques for computational biomechanics, but none of them provided a quantitative assessment of generality, robustness, automation, and accuracy in predicting strains. This study aims to quantitatively evaluate the performance of a novel mesh-morphing algorithm. A mesh-morphing algorithm based on radial-basis functions and on manual selection of corresponding landmarks on template and target was developed. The periosteal geometries of 100 femurs were derived from a computed tomography scan database and used to test the algorithm generality in producing finite element (FE) morphed meshes. A published benchmark, consisting of eight femurs for which in vitro strain measurements and standard FE model strain prediction accuracy were available, was used to assess the accuracy of morphed FE models in predicting strains. Relevant parameters were identified to test the algorithm robustness to operative conditions. Time and effort needed were evaluated to define the algorithm degree of automation.

Morphing was successful for 95\% of the specimens, with mesh quality indicators comparable to those of standard FE meshes. Accuracy of the morphed meshes in predicting strains was good $\left(R^{2}>0.9\right.$, RMSE\% < 10\%) and not statistically different from the standard meshes ( $p$-value $=0.1083)$. The algorithm was robust to inter- and intra-operator variability, target geometry refinement ( $p$-value $>0.05$ ) and partially to the number of landmark used. Producing a morphed mesh starting from the triangularized geometry of the specimen requires on average $10 \mathrm{~min}$.

The proposed method is general, robust, automated, and accurate enough to be used in bone FE modelling from diagnostic data, and prospectively in applications such as statistical shape modelling.
\end{abstract}

\section{Introduction}

A current focus of computational biomechanics research is the development of subject-specific models reproducing mechanical and geometrical features of an anatomical region of interest from biomedical images, aimed at diagnostic, therapeutic or surgical planning purposes [1]. Many techniques have already been proposed for developing finite element (FE) models of bones from computed tomography (CT) data. It seems that a mature stage of this technology has been reached with respect to in vitro model accuracy, since several independent groups have achieved very good validation results on the proximal femur loaded in vitro [2-5]. There is a substantial agreement among these validation works about how to build a model. The standard procedure for producing

\footnotetext{
* Corresponding author. Tel.: +39 051 6366554; fax: +39 0516366863.

E-mail address: schileo@tecno.ior.it (E. Schileo).
}

an accurate FE model of a bone consists first in a segmentation of the CT dataset to obtain a surface tessellation of the bone segment contour. The shape obtained is then mathematically parameterised, usually by Non-Uniform Rational B-Splines (NURBS), and finally meshed using a dedicated software. These modelling procedures, though representing the state of the art, show several limitations with respect to possible applications. A first one is automation, since they are generally user-intensive and time consuming. A second one, perhaps even more important, is flexibility, since they do not permit fast mesh adaptation and transposition between subjects and they cannot be easily used to define an indexation of the population variability in terms of both anatomical parameters and material properties distribution to generate collections of synthetic models and define response surfaces. These issues, which can globally referred to as statistical modelling, are receiving increasing attention in the literature [6-8]. Morphing (or shape registration) is a technique, originally born for computer graphics purposes, that consists in deforming a template geometry onto a 
target one [9]. It has been hypothesized to extend this technique to biomechanical applications, that is, adapting a template mesh onto a subject-specific geometry extracted from magnetic resonance (MR) or CT images [10]. Morphing of subject-specific models of bone segments from CT data is a stepping stone to explore the definition of indexation of bone shape or material properties on a population $[11,12,8]$. Moreover, it could be a promising tool to: (i) fast re-mesh when conducting sensitivity studies (e.g. on prosthesis design or positioning) (ii) easily compare results sets from two or more meshes (since morphing generates isotopological meshes, that share the same node numbering and connectivity)(iii) improve the speed and automation of subject-specific FE model generation while keeping surface regularity.

Several attempts have been already tried, based on different rationales, to relate a template to a target geometry. Couteau et al. [13] described a mesh morphing technique based on warping and local displacement functions. The algorithm was tested on 11 proximal femur geometries, one for creating the template mesh and 10 used as target geometries. The results, though described as satisfactory, were not compared to any experimental data. The same method has been applied to entire femurs and also to soft tissues, such as maxillofacial models for computer aided surgery planning and simulation, by Luboz et al. [14,15]. O'Reilly and Whyne [16] developed morphed subject-specific FE models of spinal motion segments, where mesh adaptation was based on the knowledge of some characteristic biomechanical lengths. In the absence of a comparison with experimental data, a comparison with respect to a standard FE model showed a different strain distribution in the morphed model, due to a cortical shell thicker than in the standard FE model. Tada et al. [17] generated subject-specific FE models of fingertips using a shape morphing technique where the spatial transformation was computed using a volume registration technique based on intensity gradient of MR volume data. The study focused on two specimens and there was no comparison with experimental data. Rajamani et al. [18] presented a morphing tool for patient-specific 3D knee surfaces visualization in computer assisted surgery, based on principal component analysis (PCA). The algorithm robustness was tested on 14 proximal femur specimens by means of the leave-one-out method, however the aim was not the definition of subject-specific finite element models. Hraiech et al. proposed a 3D mesh generation method for proximal femurs, consisting in the surface morphing of a template mesh constrained by manually picked landmarks [19], and the subsequent 3D mesh morphing using radial basis functions (RBF) [20]. The technique was tested on 15 specimens but only in terms of morphed mesh quality and geometrical accuracy of the morphed surface. This morphing method has been extended by using spherical instead of planar parameterisation as intermediate morphing domain [21]. Yoo [22] developed a shape morphing technique based on shape deformation method using an implicit function. This method showed good results, even if it reports local self-intersection problems when morphing complex models. However, its application to morphing bones (long bones as the femur in particular) could be difficult since it relies on the manual placement of many landmarks, that could not be repeatably identified as anatomical repere points. Sigal et al. [10] reported a comparison between two of the most frequently adopted techniques for mesh adaptation: (i) automated wrapping, guided by energy minimization criteria, that consists in mapping both the source and the target mesh on a simple geometry; (ii) manual landmarks selection, where a set of landmarks defining one to one correspondences is identified on the template mesh and on the target geometry, and morphing is achieved by making the landmark pairs coincident and applying some smoothing or non-intersection criterion to nodes other than the manually defined ones. The results showed that manual landmarks selection, though not completely automated, can ensure coincidence between source and target, while automated wrapping methods cannot.

Morphing technique has also been used for volume morphing, i.e. for the direct registration of different 3D volume data. Many algorithms have been proposed, based on different techniques such as wavelet domain-based morphing [23], geometrically constrained sphere mapping optimization [24], warping and blending [25]. To the authors' knowledge, only one work on volume morphing can be directly related to bone biomechanics modelling: [26] described the use triangularized template geometry to automatically segment a CT volume using a morphing method based on the iterative accumulation of displacement fields. However, a quantitative evaluation of the boundary recovery is lacking.

Very recently, a work of Bryan et al. [8] used morphing within a complete statistical modelling workflow for the creation of a three dimensional, statistical, finite element analysis ready model of the femur. In that work, surface morphing, taking as input a surface segmented from CT data, is based on an elastic registration scheme and volume morphing is obtained solving decoupled three-dimensional Laplace equations to deform the baseline volume mesh. Morphed meshes, after CT based material mapping, are subjected to principal component analysis to define the statistical model.

While all the cited works contributed to demonstrate the applicability of morphing techniques to biomechanics modelling, and [8] also demonstrated the feasibility of population based modelling some improvements in the morphing evaluations may be achieved. In fact, to the authors' knowledge no study evaluated morphing performances on a database characterised in terms of inter-subject anatomical variability, which can be very large, nor any study tried a comparison of the calculations resulting from morphed mesh with experimental in vitro data.

The aim of this work is to evaluate the performances of a new mesh-morphing algorithm on a large database of human femoral anatomies derived from CT scans. The proposed morphing algorithm will be evaluated in terms of generality, strain prediction accuracy, robustness and degree of automation.

\section{Materials and methods}

\subsection{Morphing procedure}

\subsubsection{Template mesh}

The template mesh was generated using the ICEM ANSYS software (Ansys, Inc., USA). Starting from a bone geometry randomly picked from the database described in Section 2.2.1.1, a tetrahedral mesh was automatically generated by the Octree meshing method [27]. The resulting mesh has an excellent element quality: average aspect ratio (AR) 1.55, maximum AR 4.73, maximum volumetric skewness of 0.60 (Fig. 1); this is a key prerequisite since it will be subsequently distorted to adapt to different shapes.

\subsubsection{Morphing}

The algorithm morphs a volumetric template mesh onto a faceted 2D specific geometry, producing a volumetric mesh of the specific geometry considered. The inputs required are a watertight femoral faceted geometry and a set of landmarks, corresponding to some relevant points of the template mesh. Usually, 10 landmarks are used (one in the femoral head, one in the lesser trochanter, two in the greater trochanter, five in the distal epiphysis around the condyles, and one in the middle of the shaft on the edge of the linea aspera) (Fig. 2). The present study adopts tetrahedral meshing, but the method can be applied independently to tetrahedra or hexahedra.

The template volume mesh is mapped to the geometry of the patient using a morphing method based on a radial basis function 


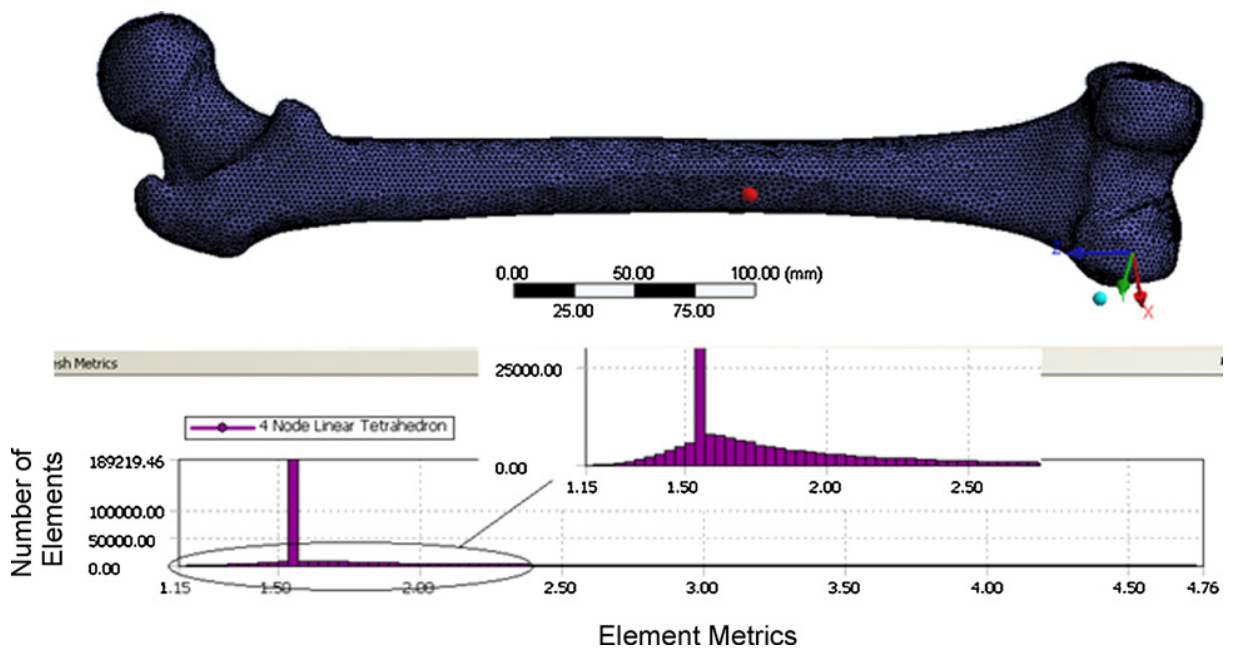

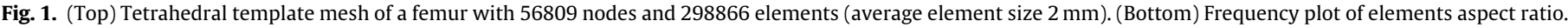

(RBF) regression, constrained by the pre-selected landmarks. The use of RBF has been reported for data smoothing, surface reconstruction, and repairing incomplete meshes [28]. The potential of RBF for morphing like applications has been demonstrated [29], i.e. for image warping applied to facial expressions [19,21] and for mesh generation. An overview of the morphing method is shown in Fig. 3, and its main steps are summarised (Fig. 4) below:

Pre-processing of the template surface mesh to be aligned to the specific geometry, by

- translating of the template mesh by aligning its centroid onto the STL centroid;

- rotating the template mesh around its shaft axis in order to put the landmarks of head and greater trochanter of template mesh on the corresponding landmarks of the STL;

- scaling the bounding box of the template mesh to the bounding box of the STL;

- extracting the surface mesh of the template.

In case a left femur is morphed on a right femur or vice versa, a mirror transformation is preliminary applied, followed by a reorientation of the mesh elements.

Surface morphing of the template surface mesh on the specific geometry, using the defined landmarks as constraints and interpolating the motion of all surface nodes based on the motion of the

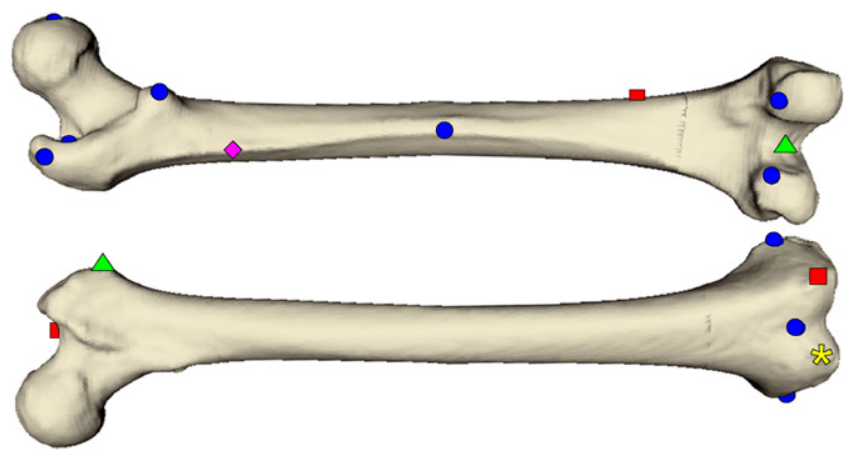

Fig. 2. Landmarks used for mesh morphing: the 10 landmarks of the original protocol (in circles, •); the 3 landmarks added for the 13 landmarks test (in squares, $\mathbf{\square}$ ); the further 3 landmarks added for the 16A test (in triangles, $\mathbf{\Delta}$ and rhombs, $\$$ ); 16B test is obtained from the $16 \mathrm{~A}$ by substituting the rhomboidal ( $)$ landmark with the asterisk $\left({ }^{*}\right)$. landmarks. Intuitively, the motion of a node should depend on its relative distance to the landmarks, such that the motion of a node close to a landmark is similar to that of the landmark, while the motion of a node far from landmarks is smoothly interpolated from the motion of all landmarks. To obtain this behaviour, a regression method based on an RBF representation was chosen, where each landmark corresponds to the centre of a basis function $k$, solving a linear equation system, which constraints the influence of motions between reference points and minimizes the deformation close to the constrained points.

Let landmarks be denoted by $p_{i}, i=1 \ldots, n$, and surface nodes by $x_{i}, i=1 \ldots, N$

Eq. (1) describes the motion of a node $x_{i}$ as the weighted sum of that of all the $n$ landmarks:

$x^{\text {new }}=f\left(x^{\text {old }}\right)=x^{\text {old }}+\sum_{i=1}^{n} k\left(x^{\text {old }}, p_{i}\right) w_{i}$

The coefficients $w_{i}$ are found using the constraint $f\left(p_{i}\right)=p_{i}^{\prime} ; i=1$, $\ldots, n$, where $p_{i}$ is the initial position of a landmark on the template mesh and $p_{i}^{\prime}$ is the final position of the corresponding landmark on the target geometry, as marked by the user.

This leads to Eq. (2):

$$
\left[\begin{array}{l}
p_{1}^{\prime}-p_{1} \\
\vdots \\
p_{i}^{\prime}-p_{i} \\
\vdots \\
p_{n}^{\prime}-p_{n}
\end{array}\right]=\left[\begin{array}{lllll}
k_{11} & \cdots & k_{1 j} & \cdots & k_{1 n} \\
\vdots & \ddots & & & \\
k_{i 1} & & k_{i i} & & \\
\vdots & & & \ddots & \\
k_{n 1} & & & & k_{n n}
\end{array}\right] \times\left[\begin{array}{l}
w_{1} \\
w_{2} \\
\vdots \\
\vdots \\
w_{n}
\end{array}\right]
$$

Or in matrix form: $P=K x W$, where $w_{i}, i=1 \ldots, n$ are the unknowns of the linear system. Having as many basis functions as constraints, matrix $K$ is square, and can be inverted to obtain $W=K^{-1} x P$.

In the present implementation of surface morphing, inverse multi-quadratic RBF were used, defined by Eq. (3):

$k(x, p)=\left(\|x-p\|^{2}+c^{2}\right)^{\beta}$

where $P$ is the centre of the basis function (i.e. a landmark); $c$ is a positive coefficient controlling the radius of the basis function (i.e. a small values of $c$ results in high local deformations and may lead to distorted elements; conversely, for larger values of $c$ the deformation is distributed over a larger region); $\beta(-1 \leq \beta \leq 0)$ is a coefficient controlling how strongly nodes that are outside the radius are weighted (i.e. the greater $\beta$ the higher the weights). In 


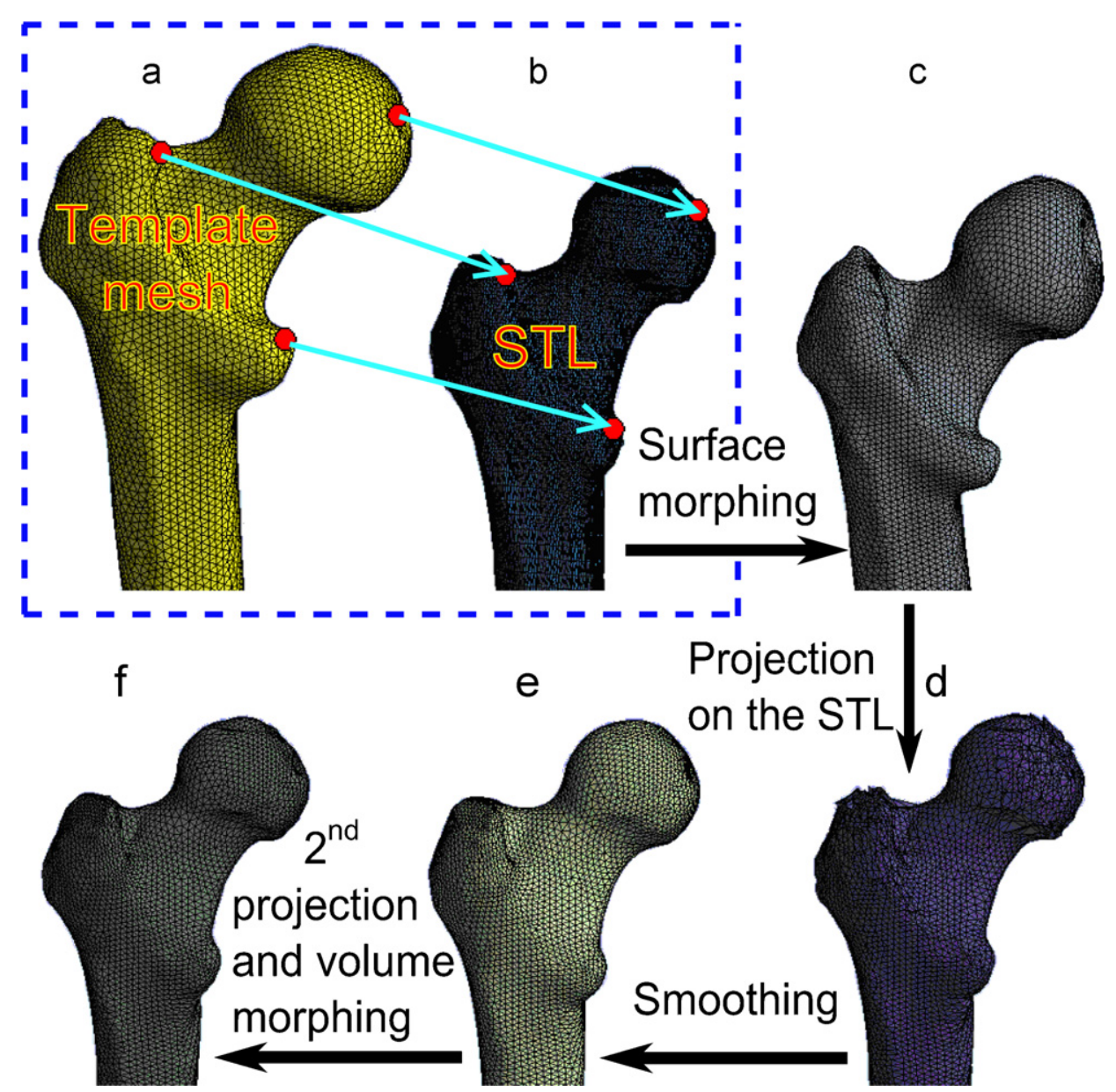

Fig. 3. Steps of the morphing algorithm (shown on the proximal femur): (a) original template mesh, (b) original STL geometry, (c) result from morphing the template mesh on the STL using RBF method constrained by 10 landmark points (given in red), (d) result after projecting (c) on (b), (e) result from the Laplacian smoothing, (f) final result represented in a high quality mesh of the STL geometry. Steps (d) and (e) are iterated as described in Section 2. (For interpretation of the references to color in this figure legend, the reader is referred to the web version of the article.)

the present study, values of $c$ and $\beta$ were chosen empirically: for each landmark $c$ was set to the distance between the its position in the template mesh and its correspondent position in the target $\mathrm{STL}, \beta$ was set to -0.15 , to optimise the results of morphing.
Projection of the resulting surface mesh on the STL geometry. Since the morphing is based on few landmarks, it results in a surface mesh isotopological with the template mesh but with a poor recovery of the STL geometry (Fig. 3(c)). To adjust it, an orthogonal projection

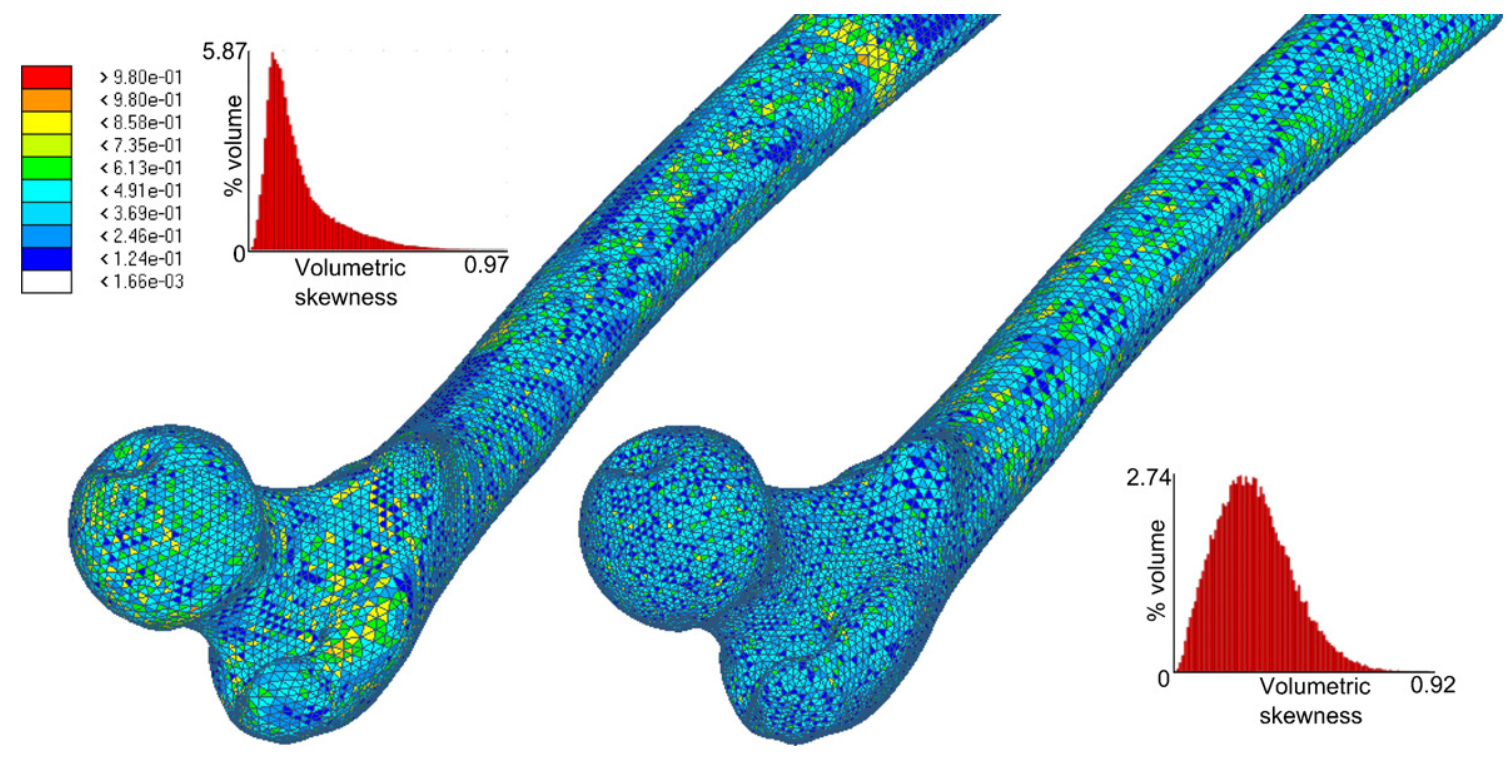

Fig. 4. Contour plot and cumulative frequency diagram of volumetric skewness for morphed mesh (left) and standard mesh (right). 
is performed, where each node in the morphed surface mesh is perpendicularly projected on the centroid of the closest triangle in the STL.

Laplacian smoothing. The projection algorithm brings to a good geometrical match but generally yields a poor quality mesh because of intersecting triangles and high aspect ratios (Fig. 3(d)). A smoothing method based on a Laplacian operator is used to correct these problems. This operator consists in replacing each node of the mesh with the centroid of its neighbouring nodes. In the proposed implementation, the Laplacian operator is applied twice, and is followed by a re-projection of the resulting surface mesh on the STL geometry, to correct the shrinkage usually induced by Laplacian smoothing. The whole process is iterated three times, since preliminary tests showed that this was sufficient to obtain a non-manifold surface with acceptable element quality.

$3 D$ morphing of the template volume mesh (outer surface nodes and inner nodes), using the nodes of the morphed surface mesh (resulting from the surface morphing after projection and Laplacian smoothing) as constraints (i.e centres of the radial basis functions). The same method of the surface morphing is applied in 3D, to define the new positions of the solid mesh nodes. In volumetric morphing Gaussian RBF were used, defined by Eq. (4):

$k(x, p)=\exp \left(-\frac{\|x-p\|^{2}}{2 \sigma^{2}}\right)$

where $p$ is the centre of the basis function, i.e. a surface node, $\sigma$ $(\sigma>0)$ is a coefficient controlling the radius of the basis function (i.e. acting on the interpolation smoothness of all nodes, inducing localised deformations for small $\sigma$ values and global deformations for high $\sigma$ values). The choice of $\sigma$ value depends on the resolution of the template mesh and on the number of surface nodes that are used for volume morphing. In the present study, it was empirically set to a low value of 0.1 to maximise element quality.

The accuracy in the boundary recovery with respect to the original STL was deemed a pre-requisite for any further evaluation of morphing. Therefore, a control was introduced in the morphing procedure to ensure that the average and maximum distance between the morphed surface mesh and the original STL were lower than $0.1 \mathrm{~mm}$ and $1 \mathrm{~mm}$ respectively. The limit values were derived from a preliminary analysis of segmentation errors from a CT dataset, where in-plane pixel spacing is typically ranging from 0.3 to $1 \mathrm{~mm} \mathrm{[30]} \mathrm{and} \mathrm{segmentation} \mathrm{precision} \mathrm{is} \mathrm{in} \mathrm{the} \mathrm{order} \mathrm{of} \mathrm{the}$ pixel size.

\subsection{Morphing evaluation procedure}

According to recommendations on methods evaluation for the application to clinical practice [27], the morphing algorithm was evaluated in terms of:

- generality of its applicability to a whole population;

- accuracy in the predictions of in vitro experimentally measured strains using the morphed meshes;

- robustness of the procedure to some operative conditions;

- automation with respect to a standard method for mesh development.

\subsubsection{Generality}

The generality of the morphing algorithm was evaluated with respect to the ability to produce usable and geometrically accurate morphed meshes for different femoral anatomies.

2.2.1.1. Database of femora. A collection of $100 \mathrm{CT}$ datasets was extracted from a database of the Rizzoli Orthopaedic Institute, originally collected for total hip replacement pre-surgical planning
Table 1

Descriptive statistics of the anatomical measurements performed on the database of femora.

\begin{tabular}{lccc}
\hline & $\begin{array}{l}\text { Mean value } \\
( \pm \mathrm{SD})\end{array}$ & $\begin{array}{l}\text { Maximum } \\
\text { value }\end{array}$ & $\begin{array}{l}\text { Minimum } \\
\text { value }\end{array}$ \\
\hline Biomechanical length [mm] & $406.1(27.9)$ & 482.5 & 355.8 \\
Neck length [mm] & $38.5(4.4)$ & 51.4 & 26.9 \\
Head radius [mm] & $21.9(1.6)$ & 25.9 & 18.5 \\
Epicondyle length [mm] & $80.5(12.8)$ & 95.8 & 69.0 \\
Anteversion angle [ ${ }^{\circ}$ ] & $12.8(9.2)$ & 45.45 & 0.6 \\
${\text { Varus/valgus angle }\left[{ }^{\circ} \text { ] }\right.}$ & $125.9(7.5)$ & 145.0 & 104.1
\end{tabular}

purposes. CT scans were performed using a Brightspeed GE Medical System computed tomography, following a published protocol [31]. CT voxel resolution ranged from $0.488 \times 0.488 \times 1.5 \mathrm{~mm}$ to $0.781 \times 0.781 \times 3 \mathrm{~mm}$. The majority of subjects for which the CT scan was available were affected by osteoarthritis or dysplasia of the hip. However, in many cases these defects were not bilateral; a surgeon selected from the database a subset of 100 femurs showing a normal anatomy.

The CT images were segmented using Amira [v4.0, Visage Imaging Inc., USA], and a polygonal geometry in stereolithography file format (STL in the following) was obtained for each specimen. All the morphing and post-processing operations were performed on a common 32 bit desktop PC (3.5 GB RAM, processor Intel core duo $2.66 \mathrm{GHz}$ ). The anatomical variability was characterized through previously proposed anatomical descriptors of the human femur. The following parameters were measured on the 3D reconstructed geometry, using an in-house software [32], and according to the specifications of the referenced studies: biomechanical length of the femoral shaft [33,34], femoral neck length [35], femoral head diameter [35], varus/valgus angle [35], anteversion angle [36] and epicondyle length (linear distance between medial and lateral epicondyle). Basic descriptive statistics of the measurements conducted on the database are reported in Table 1.

These measurements account for a large portion of the intersubject variability (limited to caucasic humans) and are in suitable agreement with the variability observed in published anatomical reports [35].

2.2.1.2. Generality metrics. Morphed meshes were evaluated for mesh quality. First, a pass/fail criterion was adopted to discriminate between usable and degenerated meshes. Morphed meshes with one or more elements showing a maximum volumetric skewness greater than 0.999 , therefore inhibiting the possibility of obtaining a FE solution were considered as degenerated. To characterise mesh quality, descriptive statistics of elements aspect ratio (AR) were obtained for all the morphed meshes, and compared to those of eight meshes generated with a standard procedure; moreover, maximum AR and skewness of morphed meshes were recorded.

\subsubsection{Accuracy}

2.2.2.1. Validation study. Volume morphed meshes were obtained for eight femoral geometries for which in vitro experimental strain measurements under six different loading conditions were available (see [4] for details about the experiment). For the same femora, a good strain prediction accuracy using a published FE modelling procedure from CT data had already been reported. This procedure includes (i) semi-automatic segmentation of CT data (Amira 3.1.1, Mercury Computer Systems Inc., USA), (ii) definition of NURBS model (Geomagic Studio v.7, Raindrop Geomagic Inc., USA), (iii) generation of 10-noded tetrahedral unstructured mesh (HyperMesh v.7, Altair Engineering, Inc., USA), (iv) inhomogeneous material properties mapping starting from the CT data (BoneMat_V3@), available at www.biomedtown.org (Taddei and co-workers [4])) and using the equation for densitometric CT cali- 
bration obtained with a phantom, the correction of the calibration as defined in [37], and the density-elasticity relationship derived by [38] on femoral neck specimens. FE analyses were performed with Ansys (v. 12.0, Ansys, Inc., USA) (see [4] for a detailed description of the modelling technique and [37] for details on the refinement of material mapping procedure). In the following, we will refer to this standard FE modelling technique as the "standard procedure", and the correlated objects will be related as "standard". It can be noted that morphing replaces steps (ii) and (iii) of the standard procedure. In order to evaluate the strain prediction accuracy of morphed meshes, the FE analyses described in [5] were replicated (same material modelling, same boundary conditions) in the eight morphed meshes.

2.2.2.2. Accuracy metrics. First, a check of the geometrical accuracy of the morphed and standard volume meshes with respect to the original STL files was performed. Nodal coordinates were extracted from the meshes and point-to-surface distance with STL facets calculated. The average, maximum positive and maximum negative discrepancies were reported.

The accuracy of morphed meshes with respect to in vitro measurements was assessed as follows: (i) a preliminary factorial ANOVA was performed to assess the effect of load case and specimen on the differences between measured and FE predicted principal strains; (ii) a linear regression between experimental and predicted strains was performed to quantify the global prediction accuracy; (iii) the root mean square (RMS) error and the peak error of FE predictions were calculated as error metrics. To compare the performance of standard and morphed models in predicting in vitro measurements, a non-parametric Wilcoxon test was performed on the correlation coefficient, the slope and the intercept of the fitted line, and the RMS error.

To directly compare the results of FE simulations on all nodes between standard and morphed meshes, the calculated nodal strains of the standard and morphed mesh were compared using the modal assurance criterion (MAC) [39]. The MAC is defined as a scalar constant relating the degree of consistency (linearity) between one modal and another reference modal vector. The MAC takes on values from zero, representing no consistent correspondence, to one, representing a consistent correspondence.

\subsubsection{Robustness}

The morphing procedure robustness was evaluated with respect to the STL refinement, the operative protocol and the inter- and intra-operator variability. Robustness was evaluated for all the cases in terms of the resulting mesh quality. Since preliminary results (see Section 3) indicated that the average values of mesh quality indicators did not differ a lot with respect to standard meshes, while few elements showed high AR or skewness, robustness evaluation was always performed on the maximum values of AR and skewness, because even a small variation of those parameters could be highly beneficial or detrimental.

2.2.3.1. STL refinement. Five STLs were randomly chosen from the entire database and decimated in order to obtain two copies of the original STL with respectively a half and a quarter of the original number of triangles. The decimated STLs obtained were then morphed and the resulting volume meshes were compared to the original volume meshes.

2.2.3.2. Operative protocol. The number of landmarks was considered the user parameter having the largest influence. As a consequence, different sized landmark sets were assessed to show the influence of this parameter on the mesh morphing. Five femur geometries were randomly chosen from the database and morphed using 10, 13 and 16 landmarks. Two protocols (16A and 16B) were devised for the 16 landmarks test. With respect to the standard procedure for landmark picking described in Section 2.1.2, three landmarks were added for the 13 landmarks test, on the lateral condyle, on the medial side of the diaphysis at $75 \%$ of the femoral biomechanical length and on the femoral neck. Other three landmarks were added for protocol 16A: one on the lateral aspect of the greater trochanter, one on the intercondylar fossa, and one on the lateral lip of the linea aspera at the proximal $25 \%$ percentile of the biomechanical length. In protocol 16B, the landmark on the linea aspera was replaced with one on the medial condyle. All the landmark locations are depicted in Fig. 2.

2.2.3.3. Inter- and intra-operator variability. Five STLs were randomly chosen and morphed by three different instructed operators performing a blind test. In addition, each operator performed the standard (10 landmarks) virtual palpation procedure three times on each STL to assess the intra-operator variability.

\subsubsection{Automation}

The automation of the morphing process was evaluated for each trial in terms of the time required to obtain a finite element mesh starting from the STL, and compared to the time necessary for creating a mesh using the standard procedure [4].

\section{Results}

\subsection{Generality}

The proposed morphing technique was able to generate nondegenerated meshes for 95 of the 100 STLs analysed. All the failed STLs presented anatomical parameters comprised in the first standard deviation from the database average. The failed elements were located in the greater trochanter region in all failed meshes.

However, morphed mesh quality was generally good, with an average AR of 1.91, equivalent to that calculated for the reference standard meshes (1.96). In all morphed meshes there were a few highly skewed elements, with a repeatable localisation in the greater trochanter region, consistently with the location of failed elements in the five degenerated meshes. The volume morphed meshes had an average maximum AR of 40 and an average maximum skewness of 0.995 . Only $44 \pm 61$ elements (i.e. less than $0.03 \%$ over the total number of elements) for each femur showed a skewness value greater than 0.98 , and $5 \pm 18$ elements (less than $0.01 \%$ ) an aspect ratio greater than 15 , being 15 and 0.98 the absolute maximum values found in the reference standard $\mathrm{FE}$ meshes.

\subsection{Accuracy}

\subsubsection{Geometrical accuracy with respect to the STL}

The average positive and negative deviations ranged from 0.09 to $0.19 \mathrm{~mm}$ (mean $0.13 \mathrm{~mm}$ ) and from -0.14 to $-0.19 \mathrm{~mm}$ (mean $-0.14 \mathrm{~mm}$ ) for the morphed meshes; from 0.03 to $0.08 \mathrm{~mm}$ (mean $0.05 \mathrm{~mm}$ ) and from -0.05 to $-0.10 \mathrm{~mm}$ (mean $-0.07 \mathrm{~mm}$ ) for the standard meshes. The maximum positive and negative deviations ranged from 0.86 to $2.72 \mathrm{~mm}$ (mean $1.33 \mathrm{~mm}$ ) and from -0.94 to -2.94 (mean $1.51 \mathrm{~mm}$ ) for the morphed meshes; from 0.56 to $1.73 \mathrm{~mm}$ (mean $1.00 \mathrm{~mm}$ ) and from -0.61 to -1.77 (mean -1.14 ) $\mathrm{mm}$ for the standard meshes. The maximum positive deviations were localised either for morphed and standard meshes in intertrochanteric fossa or fovea. The maximum negative deviations were localised in the supero-anterior part of the greater trochanter or in the lesser trochanter for morphed meshes, always in the lesser trochanter for standard meshes. Deviations exceeded the CT pixel size only in small regions around location of maxima. 
Table 2

Metrics of in vitro strain prediction accuracy for morphed and standard FE models.

\begin{tabular}{lcc}
\hline & Morphed meshes & Standard FE meshes \\
\hline$R^{2}$ & 0.94 & 0.95 \\
Slope & 0.94 & 0.97 \\
Intercept $(\mu \varepsilon)$ & 0 & -1 \\
RMSE $(\mu \varepsilon)$ & 89 & 83 \\
RMSE\% & $7.8 \%$ & $7.0 \%$ \\
Maximum error $(\mu \varepsilon)$ & 569 & 478 \\
Maximum error $\%$ & $49.0 \%$ & $42.0 \%$ \\
\hline
\end{tabular}

\subsubsection{In vitro strain prediction accuracy}

All the measurements were pooled together (factorial ANOVA: $p$-value $>0.9$ for all load cases and all specimens). A high accuracy of morphed models predictions with respect to experimental measurements was found (Table 2 ). No statistically significant differences were found between the validation results of morphed and standard models (Wilcoxon signed rank test: $p$-value $=0.1083$ ).

Comparison of strain fields between morphes and standard meshes. The MAC tests between morphed and standard mesh results yielded a MAC value greater than 0.999 for all the 8 cases.

\subsection{Robustness}

Robustness to STL refinement: the variations of the maximum AR and of the maximum skewness of the morphed meshes due to refinement of the STL surface were found statistically nonsignificant (all Wilcoxon signed rank tests: $p$-value $>0.05$ ).

Robustness to the virtual palpation procedure: a statistically significant decrease in the maximum AR and in the maximum skewness ( $p$-value $=0.04$ for both maximum AR and maximum skewness) was induced by protocol $16 \mathrm{~A}$, but not by protocol $16 \mathrm{~B}$ nor by the protocol with 13 key-points ( $p$-values 0.34 and 0.08 for the maximum skewness, $p$-values 0.14 and 0.04 for the maximum $A R$, respectively).

Inter-operator variability: no statistically significant differences were observed in the maximum AR and maximum skewness ( $p$-value $>0.05)$. Analogous results were obtained for the intraoperator variability tests.

\subsection{Automation}

An experienced operator was able to pick the landmarks in about $6 \mathrm{~min}$, following the 10 landmarks protocol described in Section 2.1.2. The time required for mesh import, pre- and post-processing (mostly depending on the hardware properties) varied between 3 and $4 \mathrm{~min}$. In summary, the average time to obtain a FE mesh from a STL geometry, using the morphing procedure, was $10 \mathrm{~min}$, with a 35 min (78\%) saving with respect to the standard procedure [4].

\section{Discussion}

The aim of the present work was to comprehensively evaluate the performance of a new meshing technique based on morphing, when applied on human femora to obtain a subject-specific FE mesh starting from a faceted geometry, such as that usually derived from the segmentation of CT scan data. The technique was evaluated by: (i) verifying its applicability on a large database of femur geometries obtained from in vivo CT data; (ii) comparing the morphed meshes with those obtained with a standard and validated meshing procedure, in terms of boundary recovery, calculated strain fields, and in vitro strain prediction accuracy; (iii) evaluating the technique robustness with respect to several operative conditions and the degree of automation of the whole process.

The morphing technique produced good results for all the indicators. As to the generality, i.e. the ability to morph a large number of STLs, some of which showed extreme anatomical conditions, the method performed well. The five STLs that failed to be morphed did not show extreme values of the anatomical parameters measured. All failed elements were in the greater trochanter region, suggesting that the morphing procedure could fail in correspondence of surface irregularities of the femoral surface, which are often present in that region and often reflected in irregularities of the STL geometry. Results can be improved through: (i) pre-processing of the STL geometries (e.g. smoothing); (ii) releasing the geometrical accuracy limit on the surface; (iii) adding landmarks in the critical regions. In fact, adding landmarks up to a total of 16 , all femurs in the database could be successfully morphed. However, one of the basic requirements that drove the development was to keep as low as possible the effort requested to the operator. Considering that while repeatable picking of 10 landmarks on a femur is generally feasible for a non-experienced operator after few instructions, finding 16 landmarks based on anatomical repere points is challenging, a default setting of 10 landmarks was kept, then relying on the flexibility of morphing implementation, that permits adding landmarks if the process fails.

With respect to element quality, few low quality elements (in terms of AR and skewness) were detected in most of the morphed meshes. All low quality elements were localised next to high curvature features and surface irregularities. The worst quality elements were repeatably placed in a region of the greater trochanter that is not critical for the purpose of the analysis for which this algorithm has been developed (stress and strain state in the bone in general, proximal femur risk of fracture in particular). Reduction of elements distortion (e.g. by using an advanced Laplacian filter such as that proposed by [40]) will anyway be a subject of future algorithm development.

However the accuracy of morphed vs. standard FE models was positively assessed. As to the accuracy in boundary recovery with respect to the original STL geometry, even if standard meshes showed lower average and maximum deviations: (i) the average deviations of both morphed and standard meshes were always well below the CT pixel size, which is a reasonable estimate of the STL precision, and (ii) the maximum deviations, fairly high for both standard and morphed meshes, were very localized (intertrochanteric fossa or fovea for the positive deviation, superoanterior part of the greater trochanter or lesser trochanter for the negative deviation), so that deviations exceeded the pixel size only in very small areas of marginal relevance (when stresses and strain on the femur are to be evaluated).

When used to predict in vitro strain measurements at various locations in the femoral head, neck and diaphysis. The morphed meshes showed an accuracy which is high and not distinguishable with that of state of the art standard FE models. The comparison of the whole strain field across the model further supported the equivalence of morphed vs. standard model results.

The morphing procedure demonstrated to be robust to the STL refinement as well as to intra- and inter-operator variability. The robustness tests indicated that using 16 landmarks instead of 10 could improve the mesh quality in terms of maximum volumetric skewness. Sixteen is around the maximum number of landmarks that could be easily picked while keeping an anatomical significance, which is necessary for repeatability. A number of 10 landmarks was deemed a reasonable trade off between automation and the need to obtain non-degenerated morphed meshes for an anatomically variegate collection of STLs. It should be investigated how the improvement of maximum mesh skewness obtained using 16 landmarks reflects on morphed mesh performance. However, this was out of the scope of this study.

The speed and automation was enhanced with respect to the standard procedure both in terms of user interaction and duration of the whole mesh generation process. This improvement in 
the automation of a subject-specific modelling procedure could be considered marginal since (i) the likely most time consuming step, i.e. CT data segmentation, is retained and (ii) recent advances in meshing make the generation of quality FE meshes directly from STL possible. However, it should be considered that additional time savings could arise from the use of morphed meshes in the whole analysis process when multiple models are involved: (i) complex sets of boundary conditions (e.g. points or areas of muscle/tendon insertions on bone, whose placement is time and effort intensive) could be morphed (ii) the post-processing phase could be simplified thanks to morphed meshes isotopology. Investigating the accuracy of morphed boundary conditions may be an interesting subject for future work: a very preliminary analysis, performed morphing one femur and comparing the morphed muscle/tendon insertions to those previously directly identified by an expert operator yielded an average error of $5 \mathrm{~mm}$, suggesting the feasibility of the process.

To the authors' knowledge no other comparable studies were conducted so far, in terms of number of the tested specimens and completeness of the evaluation procedure. In fact, previous works used a smaller number of test specimens and no evaluation of in vitro morphed models accuracy with respect to standard models was reported. Boundary recovery and mesh quality on a much smaller number of specimens and for applications different from the femur were reported in $[10,16-18]$ limited to evaluate. With respect to boundary recovery in works focusing on the femur: Bryan et al. [8] reported a mean surface registration error of $0.6 \mathrm{~mm}$ on morphed surfaces (in the present work surface boundary recovery is bounded to an average of 0.1 and a maximum of $1 \mathrm{~mm}$, as described in Section 2.1.2), but no data about the boundary recovery of the volume morphed meshes; Baldwin et al. [6] measured the root mean square difference between morphed meshes and standard meshes, obtaining on the 3 tested femora a mean value of $0.87 \pm 0.47 \mathrm{~mm}$ which is at least comparable to the one reported in the present work.

When looking at published works presenting a comparison of results between morphed and standard models: Bryan et al. [8] compared the plots of percentage proximal bone volume vs. predicted strain for morphed meshes and standard meshes, loaded in one configuration, concluding for a good agreement; Bah et al. [41] compared the maximum value of von Mises strain between morphed and standard models and performed a qualitative check of strain distribution. The present work supports with a quantitative index the feasibility of obtaining equivalent results from morphed and standard models.

As to the morphing approach, the results obtained in this work corroborate the conclusion of Sigal et al. [10] about the suitability of a fixed landmarks approach when a high geometrical accuracy is required. They also corroborate, in the authors opinion, the feasibility of morphing the quite complex femoral anatomy using a limited number of landmarks as control points.

The present study has a number of limitations. Firstly, the template mesh was created starting from a randomly chosen STL from the 100 specimens of the database, and no preliminary work on its morphology was performed. However, the random choice of the STL for the generation of the template mesh might be interpreted as an indirect proof of the robustness of the technique with respect to the anatomical variability of the STLs. Another a posteriori proof of robustness may come from the analysis of the randomly chosen template: it is a quite large femoral anatomy, and mesh degeneration due to shrinking would be more likely to arise for a large than for a small template.

Secondly, no morphing was implemented for the femoral endosteal canal. However, this limitation is softened by the high refinement of the template mesh, combined with a CT attenuationbased material properties definition. As a result, no main problems in strain prediction accuracy arose during the performed accuracy tests. However, future works will aim at overcoming this limitation by adding a control of the morphology of the endosteal canal. This may be particularly important for applications in prosthetic design or analysis, where the definition of reamed cavity and the interaction of bone and prosthetic stem is of fundamental importance.

A third limitation, related to robustness, could be represented by the lack of a convergence test on the template mesh. However, meaningful results cannot be expected from this test until when a good morphing of the endosteal canal will be developed. Moreover, the refinement level of the adopted template mesh was undoubtedly high, but still manageable in terms of mesh processing and solution time with the memory and speed resources of a common desktop PC.

A fourth limitation could be related to the small sample size with which robustness tests have been conducted. The significant effort needed to perform them was a limiting factor in this respect.

Finally, while the morphimg algorithm is quite general and can handle hexahedral node connectivity, its performance on hexahedral meshes was not tested because an high quality full hexahedral mesh of femoral anatomy could not be obtained with standard meshing algorithims.

The principal implication of this work is the possibility of using a general and validated morphing algorithm to develop a different definition of bones anatomical variability. The present study adopted anatomical parameters taken from the state of the art anatomical literature [35], based on one-dimensional measurements from planar X-ray images. The proposed morphing technique, by generating isotopological volume meshes, enables the development of bone geometry and material properties characterization based on principal component analysis, such as described in $[11,8]$. Through such an instrument, a set of parameters from 3D data would accurately represent the variation of bone morphology, with several possible developments that range from the synthesis of realistic femoral anatomies, to the definition of parameterised response surfaces of FE results.

Other future works may regard the extension of morphing to pathologically deformed bones or to bones other than the femur. The algorithm in its present implementation is likely to be applicable to deformities and to other long bones, once a meaningful set of anatomical landmarks is defined. Conversely, morphing very complex shapes such as vertebrae might require a higher number of landmarks or a different approach to overcome the manual picking.

In summary, a comprehensive evaluation of a novel morphing procedure technique demonstrated that morphing, when targeted to the specific problem of obtaining FE subject specific models of femora from CT data, can be applied to variable anatomies with no trade-off with accuracy.

\section{Acknowledgements}

The present study was partially funded by EC grant VPHOP(FP7ICT2008-223865) and Emilia Romagna Region-University Research Program 2007-2009. The authors would like to thank Martino Pani for help in the data processing.

\section{Conflict of interest statement}

This work was partially supported by the European Community (project number: FP7-ICT2008-223865; project title: The Osteoporotic Virtual Physiological Human; acronym: VPHOP); both Istituto Ortopedico Rizzoli and Ansys are partners of VPHOP project. None of the authors neither received nor will receive direct or indirect benefits from third parties for the performance of this study. 


\section{References}

[1] Viceconti M, Davinelli M, Taddei F, Cappello A. Automatic generation of accurate subject-specific bone finite element models to be used in clinical studies. J Biomech 2004;37:1597-605.

[2] Bessho M, Ohnishi I, Matsuyama J, Matsumoto T, Imai K, Nakamura K. Prediction of strength and strain of the proximal femur by a CT-based finite element method. J Biomech 2007;40:1745-53.

[3] Trabelsi N, Yosibash Z, Milgrom C. Validation of subject-specific automated p-FE analysis of the proximal femur. J Biomech 2009;42:234-41.

[4] Schileo E, Taddei F, Malandrino A, Cristofolini L, Viceconti M. Subject-specific finite element models can accurately predict strain levels in long bones. J Biomech 2007:40:2982-9.

[5] Schileo E, Taddei F, Cristofolini L, Viceconti M. Subject-specific finite element models implementing a maximum principal strain criterion are able to estimate failure risk and fracture location on human femurs tested in vitro. J Biomech 2008;41:356- 67.

[6] Baldwin MA, Langenderfer JE, Rullkoetter PJ, Laz PJ. Development of subject-specific and statistical shape models of the knee using an efficient segmentation and mesh-morphing approach. Comput Methods Programs Biomed 2009;97:232-40.

[7] Sigal IA, Whyne CM. Mesh morphing and response surface analysis: quantifying sensitivity of vertebral mechanical behavior. Ann Biomed Eng 2009;38:41-56.

[8] Bryan R, Mohan PS, Hopkins A, Galloway F, Taylor M, Nair PB. Statistical modelling of the whole human femur incorporating geometric and material properties. Med Eng Phys 2010;32:57-65.

[9] Alexa M. Recent advances in mesh morphing. Comput Graph Forum 2002;21:173-98.

[10] Sigal IA, Hardisty MR, Whyne CM. Mesh-morphing algorithms for specimenspecific finite element modeling. J Biomech 2008;41:1381-9.

[11] Belenguer Querol L, Buchler P, Rueckert D, Nolte LP, Gonzalez Ballester MA Statistical finite element model for bone shape and biomechanical properties. Med Image Comput Comput Assist Interv 2006;9:405-11.

[12] Bryan R, Mohan PS, Hopkins A, Galloway F, Taylor M, Nair PB. Statistical modelling of the whole human femur incorporating geometric and material properties. Med Eng Phys 2009;32:57-65.

[13] Couteau B, Payan Y, Lavallée S. The mesh-matching algorithm: an automatic 3D mesh generator for finite element structures. J Biomech 2000;33:1005-9.

[14] Luboz V, Chabanas M, Swider P, Payan Y. Orbital and maxillofacial computer aided surgery: patient-specific finite element models to predict surgical outcomes. Comput Methods Biomech Biomed Eng 2005;8:259-65.

[15] Luboz V, Couteau B, Payan Y. 3D finite element meshing of entire femore by using the mesh matching algorithm. In: Tripped BS, editor. Proceedings of the Transactions of the 47th Annual Meeting of the Orthopaedic Research Society. 2001. p. 522.

[16] O'Reilly MA, Whyne CM. Comparison of computed tomography based parametric and patient-specific finite element models of the healthy and metastatic spine using a mesh-morphing algorithm. Spine (Phila Pa 1976) 2008;33:1876-81.

[17] Tada M, Yoshida H, Mochimaru M. Geometric modeling of living tissue for subject-specific finite element analysis. Conf Proc IEEE Eng Med Biol Soc 2006;(Suppl.):6639-42.

[18] Rajamani KT, Hug J, Nolte LP, Styner M. Bone morphing with statistical shape models for enhanced visualization. In: Medical Imaging 2004: Visualization, Image-Guided Procedures, and Display. 1st ed. San Diego, CA, USA: SPIE; 2004 p. 122-30.

[19] Hraiech N, Taddei F, Malvesin E, Rochette M, Viceconti M. Fast 3D mesh generation of femur based on planar parameterization and morphing. In: 5th IEEE International Symposium on Biomedical Imaging: From Nano to Macro, 2008. ISBI. 2008. p. 1561-4.
[20] Bishop CM. Pattern recognition and machine learning. Springer; 2006 p. 740.

[21] Hraiech N, Rochette M, Taddei F, Viceconti M. Finite element femur generation using morphing and spherical parameterization techniques. In: Book of Abstracts: IV International Congress on Computational Bioengineering including the first European Symposium on Biomedical Integrative Research: DEIS, Alma Mater Studiorum. 2009. p. 27.

[22] Yoo D-J. Three-dimensional morphing of similar shapes using a template mesh. Int J Precision Eng Manuf 2009;10:55-66.

[23] Taosong H, Wang S, Kaufman A. Wavelet-based volume morphing. In: IEEE Conference on Visualization, 1994, Visualization '94 Proceedings. 1994. p. 85-92. CP8.

[24] Athanasiadis T, Fudos I, Christophoros N, Vasiliki S. Feature-based 3D morphing based on geometrically constrained sphere mapping optimization. In: Proceedings of the 2010 ACM Symposium on Applied Computing. Sierre, Switzerland: ACM; 2010

[25] Lin L, Lee C, Lee T-Y. Distributed volume morphing. Cluster Comput 1999;2:219-27.

[26] Pettersson J, Borga M, Andersson M, Knutsson H. Volume morphing for segmentation of bone from 3D data. In: Symposium on Image Analysis SSBA. 2005.

[27] Viceconti M, Taddei F. Automatic generation of finite element meshes from computed tomography data. Crit Rev Biomed Eng 2003;31:27-72.

[28] Carr J, Beatson R, Cherrie J, Mitchell T, Fright R, McCallum B, et al. Reconstruction and representation of 3D objects with radial basis functions. In: Fiume E, editor. SIGGRAPH 2001, Computer Graphics Proceedings. ACM Press/ACM SIGGRAPH; 2001. p. 67-76.

[29] Arad N, Dyn N, Reisfeld D, Yeshurun Y. Image warping by radial basis functions: applications to facial expressions. CVGIP: Graph. Models Image Process 1994;56:161-72.

[30] Taddei F, Martelli S, Reggiani B, Cristofolini L, Viceconti M. Finite-element modeling of bones from CT data: sensitivity to geometry and material uncertainties. IEEE Trans Biomed Eng 2006;53:2194-200.

[31] Viceconti M, Lattanzi R, Antonietti B, Paderni S, Olmi R, Sudanese A, et al. CT-based surgical planning software improves the accuracy of total hip replacement preoperative planning. Med Eng Phys 2003;25:371-7.

[32] Viceconti M, Taddei F, Montanari L, Testi D, Leardini A, Clapworthy G, et al Multimod data manager: a tool for data fusion. Comput Methods Program Biomed 2007;87:148-59.

[33] Cristofolini L. A critical analysis of stress shielding evaluation of hip prostheses. Crit Rev Biomed Eng 1997;25:409-83.

[34] Ruff CB, Hayes WC. Cross-sectional geometry of Pecos Pueblo femora and tibiae-a biomechanical investigation. II. Sex, age, side differences. Am J Phys Anthropol 1983;60:383-400.

[35] Noble PC, Box GG, Kamaric E, Fink MJ, Alexander JW, Tullos HS. The effect of aging on the shape of the proximal femur. Clin Orthop Relat Res 1995:31-44.

[36] Murphy SB, Simon SR, Kijewski PK, Wilkinson RH, Griscom NT. Femoral anteversion. J Bone Joint Surg Am 1987;69:1169-76.

[37] Schileo E, Dall'ara E, Taddei F, Malandrino A, Schotkamp T, Baleani M, et al. An accurate estimation of bone density improves the accuracy of subject-specific finite element models. J Biomech 2008;41:2483-91.

[38] Morgan EF, Bayraktar HH, Keaveny TM. Trabecular bone modulus-density relationships depend on anatomic site. J Biomech 2003;36:897-904.

[39] Allemang RJ, Brown DL. A correlation coefficient for modal vector analysis. In: International Modal Analysis Conference. 1982. p. 110-6.

[40] Vollmer J, Mencl R, uuml ller H. Improved Laplacian smoothing of noisy surface meshes. Comput Graph Forum 1999;18:131-8.

[41] Bah MT, Nair PB, Browne M. Mesh morphing for finite element analysis of implant positioning in cementless total hip replacements. Med Eng Phys 2009;31:1235-43. 